\title{
BRANCH POINTS OF SOLUTIONS OF EQUATIONS IN BANACH SPACE. II $\left.{ }^{(}\right)$
}

\author{
BY \\ JANE CRONIN
}

1. Introduction. In previous papers [2] and $[3]\left({ }^{2}\right)$, a multiplicity for the local solutions of an abstract functional equation was defined and studied. The theory developed was used to obtain existence theorems for integral equations and elliptic differential equations. The purpose of this paper is to extend the multiplicity theory and apply the new results to obtain more existence theorems for integral and differential equations.

We study the equation in a Banach space $\mathfrak{X}$,

$$
(I+C+T) x=y
$$

where $x, y \in \mathfrak{X}, I$ is the identity transformation, $C$ is linear and completely continuous, and $T$ is a higher order transformation such that $T(0)=0$. (Transformation $T$ is not, in general, completely continuous.) The problem is to investigate the solutions $x$ near 0 of (1.1) for given $y$ sufficiently close to 0 . In $\S 2$, we recall the definition of multiplicity $\left({ }^{3}\right)$ and show that the multiplicity of the solution $x=0$ of (1.1) with $y=0$ is defined if and only if $x=0$ is an isolated solution of (1.1) with $y=0$ (Theorem 2.1). We also show (Theorem 2.3) that if $I+C+T$ is a 1-1 mapping, the multiplicity is +1 or -1 .

We apply Theorem 2.3 in $\$ 4$ to obtain an existence theorem for a certain class of integral equations and in $\$ 5$ to obtain a new proof of a theorem of Schauder [8, Satz IV] for elliptic differential equations by showing that the integral and differential equations are special cases of (1.1).

The new proof of Schauder's theorem uses simpler topological considerations than those used by Schauder. Also this proof fits Schauder's theorem into a wider picture of the elliptic differential equation. For by using the multiplicity theory, we are able to treat the case in which there are branchings of the solutions of the differential equation. The multiplicity is defined in terms of topological index and hence gives no direct information about the number of distinct solutions of (1.1) for given $y$. However if the transformation $T$ has a differential and if the multiplicity $m$ is different from zero, then in each neighborhood of $y=0$, there is an infinite set $Q$ such that for each $y \in Q$, equation (1.1) has at least $|m|$ distinct solutions $x$ near 0 (Theorem

Received by the editors April 7, 1951 and, in revised form, December 12, 1952.

(1) Most of this was done while the writer was on a Horace H. Rackham Postdoctoral Fellowship from the University of Michigan.

(2) Numbers in brackets refer to the bibliography at the end of the paper.

(3) The definition given here is a simplified version of the definition given in [2] and [3]. 
3.1). Thus if $|m|>1$, there are actual "branchings" of the solutions of (1.1). This is proved by using a theorem due to A. Sard [7].

In $\S \S 4$ and 5 , it is shown that certain integral equations and the elliptic differential equations studied by Schauder in [8] and further studied in [3] are examples of equation (1.1). Hence Theorem 3.1 shows that for such equations, there are "branchings" of the solutions.

2. The multiplicity. Let $\mathfrak{X}$ be a Banach space and 0 its zero. We consider the equation

$$
(I+C+T) x=y
$$

where $x, y \in \mathfrak{X}, I$ is the identity transformation, $C$ is linear and completely continuous, and $T$. is a continuous transformation of $\mathfrak{X}$ into itself which satisfies the following conditions:

$\left(\mathrm{P}_{1}\right) T(0)=0$.

$\left(\mathrm{P}_{2}\right)$ There exist a neighborhood $\left.{ }^{4}\right) N$ of 0 and a positive constant $B$ such that $u, v \in N$ implies

$$
\|T(u)-T(v)\| \leqq B[\|u\|+\|v\|][\|u-v\|] .
$$

Clearly, $x=0$ is a solution of (2.1) when $y=0$. We define a multiplicity for this solution. If $(I+C)$ is nonsingular, i.e., if $(I+C) z=0$ implies $z=0$, then the inverse $(I+C)^{-1}$ exists according to the Riesz theory of completely continuous transformations [6, Satz 7]. Because of conditions $\left(\mathrm{P}_{1}\right)$ and $\left(\mathrm{P}_{2}\right)$, the Hildebrandt-Graves implicit function theorem [4, pp. 134-135] can be used to prove that for each $y$ sufficiently close to 0 , equation (2.1) has a unique local solution $x$. More precisely, there exist neighborhoods $N(y)$ and $N(x)$ such that for each $y_{0} \in N(y)$, there is a unique $x \in N(x)$ satisfying the equation

$$
(I+C+T) x=y_{0} .
$$

(See [2] for the proof.) In this case, we say that the multiplicity of the solution $x=0$ of (2.1) with $y=0$ is one.

Now suppose that $(I+C)$ is singular, i.e., that $(I+C) z=0$ for some $z \neq 0$. In order to define the multiplicity, we make use of some facts from the Riesz theory [6]. There is an integer $m$ (the Riesz index of $(I+C)$ ) and a direct sum decomposition

$$
\mathfrak{X}=\mathfrak{X}_{m} \oplus \mathfrak{X}^{m}
$$

such that $\mathfrak{X}_{m}$ is the finite-dimensional null space of $(I+C)^{m}$ and $(I+C) / \mathfrak{X}^{m}$ is a 1-1 transformation of the complete subspace $\mathfrak{X}^{m}=(I+C)^{m} \mathfrak{X}$ onto itself. Define the transformation $R$ as follows: select a basis for $\mathfrak{X}_{m}$ and let $A$ be the corresponding matrix representation of $(I+C) / \mathfrak{X}_{m} ; R / \mathfrak{X}_{m}$ is the matrix

(4) Unless otherwise noted, all neighborhoods are spherical with center 0 . 
which transforms $A$ into its Hermite canonical form [5, pp. 35-37] and $R / \mathfrak{X}^{m}=\left[(I+C) / \mathfrak{X}^{m}\right]^{-1}$. Transformation $R$ has an inverse and hence is a homeomorphism. It is easy to show that for $x \in \mathfrak{X}$,

$$
R(I+C) x=x-E_{1}(x),
$$

where $E_{1}$ is a projection of $\mathfrak{X}$ onto $\mathfrak{X}_{1}$, the null space of $(I+C)$. Multiplying (2.1) by $R$, we obtain:

$$
x^{1}+R T\left(x_{1}+x^{1}\right)=R(y),
$$

where $x_{1}=E_{1}(x)$ and $x^{1}=x-x_{1}$. Now multiply $(2.2)$ by $E_{1}$ and $E^{1}=I-E_{1}$ respectively:

$$
\begin{gathered}
E_{1} R T\left(x_{1}+x^{1}\right)-E_{1} R(y)=0, \\
x^{1}+E^{1} R T\left(x_{1}+x^{1}\right)-E^{1} R(y)=0 .
\end{gathered}
$$

Since $R$ has an inverse, then for given $y$, the study of the solutions $x$ of (2.1) is equivalent to the study of the simultaneous solutions $x_{1}$ and $x^{1}$ of (2.3) and (2.4).

Because of conditions $\left(\mathrm{P}_{1}\right)$ and $\left(\mathrm{P}_{2}\right)$ on $T$, the Hildebrandt-Graves theorem may be used to solve (2.4) uniquely for $x^{1}$ in terms of $x_{1}$ and $y$ if $x^{1}, x_{1}$, and $y$ are sufficiently small. That is, there exist neighborhoods $U(y), U\left(x_{1}\right)$, and $U\left(x^{1}\right)$ in the spaces $\mathfrak{X}, \mathfrak{X}_{1}$, and $\mathfrak{X}^{1}=E^{1}(\mathfrak{X})$ and a continuous function $F\left(x_{1}, y\right)$ such that if $\left(x_{1}, y\right) \in\left[U\left(x_{1}\right)\right] \times[U(y)]$, then

$$
x^{1}=F\left(x_{1}, y\right)
$$

is the unique solution in $U\left(x^{1}\right)$ of (2.4). Substituting in (2.3), we obtain:

$$
E_{1} R T\left[x_{1}+F\left(x_{1}, y\right)\right]-E_{1} R(y)=0 .
$$

Thus solving (2.1) for $x$ when given $y \in U(y)$ is equivalent to solving (2.6) for $x_{1}$.

If $y=0$, equation (2.6) has the solution $x_{1}=0$. The topological index $\left[1\right.$, p. 470] at 0 of the map $E_{1} R T\left[x_{1}+F\left(x_{1}, 0\right)\right]$ is a measure of the multiplicity of this solution. This leads to the following definition.

Definition 2.1. If $(I+C)$ is nonsingular, the multiplicity of the solution $x=0$ of (2.1) with $y=0$ is one. If $(I+C)$ is singular, the multiplicity is the topological index at 0 of the map

$$
G\left(x_{1}\right)=E_{1} R T\left[x_{1}+F\left(x_{1}, 0\right)\right] .
$$

(Hereafter the term multiplicity will mean the multiplicity of the solution $x=0$ of $(2.1)$ with $y=0$.)

THEOREM 2.1. The multiplicity is defined if and only if $x=0$ is an isolated solution of (2.1) with $y=0$, i.e., if and only if there exists a neighborhood $N_{0}$ in $\mathfrak{X}$ such that if $x \in N_{0}$, then $(I+C+T) x=0$ implies $x=0$. 
Proof. The derivation of (2.6) shows that $x=0$ is an isolated solution of (2.1) with $y=0$ if and only if $x_{1}=0$ is an isolated solution of (2.6) with $y=0$, i.e., an isolated solution of the equation: $G\left(x_{1}\right)=0$. But this is the necessary and sufficient condition that the topological index at 0 of $G\left(x_{1}\right)$ be defined.

THEOREM 2.2. If the multiplicity is different from zero, there exist neighborhoods $V(x), V(y)$ such that for each $y \in V(y)$, equation (2.1) has at least one solution $x$ in $V(x)$.

Proof. Follows from the fundamental properties of the topological index [1, Deformationssatz, p. 424 and Satz 1, p. 467].

REMARK. If the topological index at 0 of $G\left(x_{1}\right)$ is not defined, i.e., if there are points $x_{1}$ arbitrarily close to 0 for which $G\left(x_{1}\right)=0$, equation (2.6) may or may not have solutions for given $y$. If for some $y$, the topological degree at 0 of the map

$$
E_{1} R T\left[x_{1}+F\left(x_{1}, y\right)\right]-E_{1} R(y)
$$

relative to a neighborhood $S\left(^{5}\right)$ is not defined, (2.6) has at least one solution $x_{1}$ on the boundary of $S$. If the topological degree is defined and different from zero, (2.6) has a solution in $S$. If the degree is zero, (2.6) may or may not have a solution in $S$.

THEOREM 2.3. If $I+C+T$ is 1-1 in some neighborhood $N_{1}$, the multiplicity is +1 or -1 .

Proof. It suffices to prove that $G\left(x_{1}\right)$ is a homeomorphism in some neighborhood. The theorem then follows from [1, Satz VII, p. 475]. Choose the $U\left(x_{1}\right), U\left(x^{1}\right)$, and $U(y)$ used in the derivation of (2.6) so that

$$
\left[U\left(x_{1}\right)\right] \oplus\left[U\left(x^{1}\right)\right] \subset N_{1} \text {. }
$$

(The Hildebrandt-Graves theorem permits choice of arbitrarily small neighborhoods.) Let $V$ be a neighborhood in $\mathfrak{X}_{1}$ such that $R^{-1}[V] \subset U(y)$. Since $G$ is continuous, there is a neighborhood $W$ in $\mathfrak{X}_{1}$ such that $G(\bar{W}) \subset V$. Now $G\left(x_{1}\right)$ is $1-1$ on $\bar{W}$. For suppose there exist $x_{11}, x_{12} \in \bar{W}, x_{11} \neq x_{12}$, and

$$
G\left(x_{11}\right)=G\left(x_{12}\right)=z_{1} \in V .
$$

Since $V \subset R[U(y)]$, there exists $y_{0} \in U(y)$ such that $E_{1} R\left(y_{0}\right)=z_{1}$ and $E^{1} R\left(y_{0}\right)$ $=0$. Hence (2.8) may be written

$$
E_{1} R T\left[x_{11}+F\left(x_{11}, E^{1} R\left(y_{0}\right)\right)\right]-E_{1} R\left(y_{0}\right)=0
$$

and

$$
E_{1} R T\left[x_{12}+F\left(x_{12}, E^{1} R\left(y_{0}\right)\right)\right]-E_{1} R\left(y_{0}\right)=0 .
$$

(5) When we speak of the topological degree of a mapping $f$ relative to a set $M$, we mean the topological degree of the mapping $f / \vec{M}$. 
Then writing $x_{1}^{1}=F\left(x_{11}, E^{1} R\left(y_{0}\right)\right)$ and $x_{2}^{1}=F\left(x_{12}, E^{1} R\left(y_{0}\right)\right)$, we have

$$
(I+C+T)\left(x_{11}+x_{1}^{1}\right)=y_{0}
$$

and

$$
(I+C+T)\left(x_{12}+x_{2}^{1}\right)=y_{0} .
$$

This contradicts the hypothesis. So $G$ is $1-1$, continuous and hence a homeomorphism on $\bar{W}$ [1, Satz III, p. 95].

Corollary 2.1. (Invariance of Domain). Let $M$ be a 1-1 map of an open set $\mathfrak{U} \subset \mathfrak{X}$ into $\mathfrak{X}$. Suppose that for each $x_{0} \in \mathfrak{U}$, there is a neighborhood $N\left(x_{0}\right)$ of $x_{0}$ and transformations $C_{0}, T_{0}$ so that for $x \in N\left(x_{0}\right)$,

$$
M(x)=M\left(x_{0}\right)+I\left(x-x_{0}\right)+C_{0}\left(x-x_{0}\right)+T_{0}\left(x-x_{0}\right)
$$

where $I$ is the identity transformation, $C_{0}$ is linear and completelycontinuous, and $T_{0}$ is a map of the open set

$$
N_{0}=\left[y \mid y=x-x_{0} \text { where } x \in N\left(x_{0}\right)\right]
$$

into $\mathfrak{X}$ with the properties:

(1) $T_{0}(0)=0$.

(2) There is a positive constant $B_{0}$ such that $u, v \in N_{0}$ implies

$$
\|T(u)-T(v)\| \leqq B_{0}[\|u\|+\|v\|][\|u-v\|] .
$$

Then $M(\mathfrak{U})$ is an open set in $\mathfrak{X}$.

REMARK. The statement of Corollary 2.1 (and Theorems 4.2 and 5.2 which are applications in $\S \S 4$ and 5 of Theorem 2.3) does not involve the notion of multiplicity at all.

3. Branchings of the solutions. We consider the question: if the multiplicity is known, what can be said about the number of distinct solutions of equation (2.1)? If the multiplicity is zero, little can be said beyond the statement that there is no neighborhood $U(y)$ for each point of which (2.1) has a unique solution. (For if such a $U(y)$ existed, the multiplicity would be \pm 1 by Theorem 2.3.) For a given $y$, equation (2.1) may have no solutions, a unique solution, or more than one solution.

But if the multiplicity $m$ is different from zero, (2.1) has at least one solution for each sufficiently small $y$ by Theorem 2.2 . In some cases, $(2.1)$ has at least $|m|$ distinct solutions. To prove this, we put the following additional hypothesis on $T$.

$\left(\mathrm{P}_{7}\right)\left(^{6}\right)$ At each point $x$ in some neighborhood of 0 , transformation $T$ has a differential $L_{x}(\Delta x)$ which satisfies a Lipschitz condition in $x$. That is, there

(6) Conditions $\left(\mathrm{P}_{3}\right),\left(\mathrm{P}_{4}\right),\left(\mathrm{P}_{8}\right)$, and $\left(\mathrm{P}_{6}\right)$, which will not be discussed here, are conditions used in computing the multiplicity. See [3, pp. 110-111]. 
is a neighborhood $N_{d}$ such that for each $x \in N_{d}$ there is a bounded linear transformation $L_{x}$ and a transformation $Q_{x}$ both taking $\mathfrak{X}$ into $\mathfrak{X}$ and satisfying the following conditions:

$\left(\mathrm{d}_{1}\right)$ For each $\Delta x \in \mathfrak{X}, T(x+\Delta x)-T(x)=L_{x}(\Delta x)+Q_{x}(\Delta x)$.

$\left(\mathrm{d}_{2}\right)$ There is a positive constant $K$ such that $u, v \in N_{d}$ implies

$$
\left\|L_{u}-L_{v}\right\| \leqq K\|u-v\| .
$$

$\left(\mathrm{d}_{3}\right) \lim _{\Delta x \rightarrow 0} Q_{x}(\Delta x) /\|\Delta x\|=0$.

To simplify the statement of the theorem, we make the following definition.

Definition 3.1. Let $\mathcal{N}=\mathcal{N}_{1} \oplus \mathcal{N}^{1}$ where $\mathcal{N}_{1}, \mathcal{N}^{1}$ are neighborhoods in $\mathfrak{X}_{1}, \mathfrak{X}^{1}$ respectively. Suppose that corresponding to each point $x_{\nu}^{1} \in \mathcal{N}^{1}$, there is a set $E_{\nu} \subset \mathcal{N}_{1}$ of $n$-dimensional measure zero. (The $n$ is the dimension of $\mathfrak{X}_{1}$ regarded as a real Euclidean space.) A set $A \subset \mathcal{N}$ is said to contain a good many of the points of $\mathcal{N}$ if

$$
A \supset \bigcup_{v}\left[x_{v}^{1} \oplus\left(\mathcal{N}_{1}-E_{v}\right)\right] .
$$

If $h$ is a homeomorphism of $\mathfrak{X}$ onto itself, $h(A)$ is said to contain a good many of the points of the open set $h(\mathcal{N})$.

TheOREM 3.1. If the multiplicity $m$ is different from zero, there exist neighborhoods $\mathcal{N}(x)$ and $\mathcal{N}(y)$ such that for a good many of the points $y \in \mathcal{N}(y)$, the equation

$$
(I+C+T) x=y
$$

has at least $|m|$ distinct solutions in $\mathrm{N}(x)$.

Proof. In order to prove this theorem, we make use of a theorem due to A. Sard [7, Theorem 4.1]. First two definitions.

Definition 3.2. Let

$$
J:\left(x_{1}, \cdots, x_{n}\right) \rightarrow\left(x_{1}^{\prime}, \cdots, x_{n}^{\prime}\right)
$$

be a map of a region $R$ of a real Euclidean $n$-space $R^{n}$ into $R^{n}$. If $J$ can be described by equations

$$
f_{i}\left(x_{1}, \cdots, x_{n}\right)=x_{i}^{\prime} \quad(i=1, \cdots, n)
$$

such that at each point of $R$ the derivatives $\partial f_{i} / \partial x_{j}(i, j=1, \cdots, n)$ exist and are continuous, then $J$ is said to be a differentiable map in $R$.

Definition 3.3. Let $J$ be a differentiable map of region $R \subset R^{n}$ into $R^{n}$. A critical value of $J$ is the image under $J$ of a point at which the Jacobian of $J$ is zero, i.e., a critical value is the image of a critical point.

The special case of Sard's theorem that we use is this: 
LeмmA 3.1. Let $J$ be a differentiable map of a region $R \subset R^{n}$ into $R^{n}$. Then the set of critical values of $J$ has $n$-dimensional measure zero.

We use Lemma 3.1 to prove the following lemma.

LеммA 3.2. Let $J$ be a continuous map defined on the closure $\mathrm{Cl}(\mathrm{R})$ of region $R \subset R^{n}$ and differentiable in $R$. Suppose the topological degree of $J$ at point $p_{0}$ is $d \neq 0$. Then there is a neighborhood $U$ of $p_{0}$ and a set $E$ of $n$-dimensional measure zero, $E \subset U$, such that $p \in U-E$ implies that $J^{-1}(p)$ is a finite set consisting of at least $|d|$ points.

Proof. By the Deformationssatz [1, p. 424], there is a neighborhood $U$ of $p_{0}$ such that the topological degree of $J$ is $d$ at each point $p \in U$. By Lemma 3.1 , the set $E$ of points in $U$ which are critical values of $J$ has $n$-dimensional measure zero. We show that each point $p \in U-E$ is the image under $J$ of at least $|d|$ distinct points in $R$.

First $p \in J(R)$ because the topological degree at $p$ is $d \neq 0$. The set $J^{-1}(p)$ must be a set of isolated points, hence a finite set. For if there is a point $q_{0}$ which is a limit point of $J^{-1}(p)$, then $q_{0} \in J^{-1}(p)$ and so the Jacobian of $J$ at $q_{0}$ must be different from zero. But then $J$ would be a 1-1 mapping in some neighborhood of $q_{0}$ which contradicts the fact that $q_{0}$ is a limit point of $J^{-1}(p)$. Since the Jacobian of $J$ is nonzero on $J^{-1}(p)$, the topological index of $J$ at each $q \in J^{-1}$ is +1 or -1 . But the topological degree of $J$ at $p$ is the sum of the indices of $J$ at the points of $J^{-1}(p)$ [1, Satz II, p. 472]; so there must be at least $|d|$ distinct counterimages of $p$.

Now we are ready to prove Theorem 3.1. We choose the $U\left(x_{1}\right), U\left(x^{1}\right)$, and $U(y)$ used in the derivation of (2.6) so that $U(y)$ and $\left[U\left(x_{1}\right)\right] \oplus\left[U\left(x^{1}\right)\right]$ are contained in $N \cap N_{d} \cap N_{0}$ where $N$ and $N_{d}$ are the neighborhoods described in $\left(\mathrm{P}_{2}\right)$ and $\left(\mathrm{P}_{7}\right)$ and $N_{0}$ is the isolation neighborhood described in Theorem 2.1. By the same argument as for the proof of Theorem 2.2, there is a neighborhood $\mathcal{V}\left(x_{1}\right)$ in $\mathfrak{X}_{1}$ and a neighborhood $\mathcal{V}(y)$ in $\mathfrak{X}$ such that for each $y \in \mathcal{V}(y)$, the topological degree at 0 and relative to $\mathcal{V}\left(x_{1}\right)$ of the map

$$
E_{1} R T\left[x_{1}+F\left(x_{1}, y\right)\right]-E_{1} R(y)
$$

is $m$.

First we prove the theorem using the assumption that for each $y \in \mathcal{V}(y)$, the map (3.1) is a differentiable map in $\nu\left(x_{1}\right)$. Then we complete the actual proof of the theorem by proving this assumption.

We return to (2.5), the Hildebrandt-Graves solution $x^{1}$ of equation (2.4). Clearly, $x^{1}$ is a function of $x_{1}$ and $E^{1} R(y)$, i.e.,

$$
x^{1}=H\left(x_{1}, E^{1} R(y)\right)=F\left(x_{1}, y\right) .
$$

Let $\mathcal{V}^{1}$ and $\mathcal{V}_{1}$ be neighborhoods in $\mathfrak{X}^{1}$ and $\mathfrak{X}_{1}$ respectively such that

$$
R^{-1}\left[\mathcal{U}^{1} \oplus \mathcal{U}_{1}\right] \subset \mathcal{V}(y) .
$$


Then the topological degree at zero and relative to $\nu\left(x_{1}\right)$ of the map

$$
E_{1} R T\left[x_{1}+H\left(x_{1}, u^{1}\right)\right]-u_{1}
$$

is $m$ for all $u^{1}+u_{1} \in V^{1} \oplus V_{1}$. From this, it follows that the degree at arbitrary $u_{1} \in V_{1}$ of $E_{1} R T\left[x_{1}+H\left(x_{1}, u^{1}\right)\right]$ is $m$ for all $u^{1} \in V^{1}$. Take a fixed $u_{0}^{1} \in V^{1}$. By Lemma 3.2, there is a set $E \subset V_{1}, E$ of $n$-dimensional measure zero, such that $p \in V_{1}-E$ implies that the equation

$$
E_{1} R T\left[x_{1}+H\left(x_{1}, u_{0}^{1}\right)\right]=p
$$

has at least $|m|$ distinct solutions in $\mathcal{V}\left(x_{1}\right)$. Since $R$ is a homeomorphism, then $R^{-1}\left[V^{1} \oplus V_{1}\right]$ is the desired open set $\mathcal{N}(y)$.

Now we show that for each fixed $y \in \mathcal{V}(y)$, the map (3.1) is a differentiable map in $\mathcal{V}\left(x_{1}\right)$. The constant term $E_{1} R(y)$ can be disregarded. It is sufficient to consider the case when $\mathfrak{X}_{1}$ is one-dimensional. (The proof in the general case is almost the same except that the notation is more complicated.) We write the elements of $\mathfrak{X}_{1}$ as a $\zeta$ where $\zeta$ is a fixed nonzero vector in $\mathfrak{X}_{1}$ such that $\|\zeta\| \leqq 1$. Then the problem is to prove that the real-valued function $E_{1} R T[a \zeta+F(a \zeta, y)]$ has a continuous derivative with respect to $a$.

Lemma 3.3. Suppose there is a neighborhood $\mathfrak{W}$ of $a=0$ such that for $a \in W$

$$
\lim _{\Delta a \rightarrow 0} \frac{F[(a+\Delta a) \zeta, y]-F[a \zeta, y]}{\Delta a}=\mathcal{D}(a, y)
$$

exists and is continuous in a. Then the derivative

$$
\frac{d}{d a}\left\{E_{1} R T[a \zeta+F(a \zeta, y)]\right\}
$$

exists and is continuous at each point $a \in W$.

Proof. By condition $\left(\mathrm{P}_{7}\right)$, we have:

$$
\begin{aligned}
& \frac{E_{1} R T[(a+\Delta a) \zeta+F((a+\Delta a) \zeta, y)]-E_{1} R T[a \zeta+F(a \zeta, y)\rfloor}{\Delta a} \\
&=\frac{E_{1} R L_{a \zeta+F(a \zeta, y)}[\Delta a \zeta+F((a+\Delta a) \zeta, y)-F(a \zeta, y)]}{\Delta a} \\
&+\frac{E_{1} R Q_{a \zeta+F(a \zeta, y)}[\Delta a \zeta+F((a+\Delta a) \zeta, y)-F(a \zeta, y)]}{\Delta a} \\
&= E_{1} R L_{a \zeta+F(a \zeta, y)}\left[\zeta+\frac{F((a+\Delta a) \zeta, y)-F(a \zeta, y)}{\Delta a}\right] \\
&+\frac{E_{1} R Q_{a \zeta+F(a \zeta, y)}[\Delta a \zeta+F((a+\Delta a) \zeta, y)-F(a \zeta, y)\rfloor}{\Delta a} .
\end{aligned}
$$


From $\left(d_{3}\right)$ of $\left(P_{7}\right)$ and the hypothesis of the lemma, it follows that the limit, as $\Delta a$ approaches zero, of the second term is zero. Hence

$$
\frac{d}{d a}\left\{E_{1} R T[a \zeta+F(a \zeta, y)]\right\}=E_{1} R L_{a \zeta+F(a \zeta, y)}[\zeta+\mathcal{D}(a, y)] .
$$

To complete the proof of Theorem 3.1, it is sufficient to show that the the limit (3.2) exists and is continuous in $a$. This result is a special case of a much more general theorem due to Hildebrandt and Graves [4, Theorem 4]. We describe briefly a proof of the statement needed here partly because for this special case the proof can be made quite short but mainly so that we may indicate precisely the limitations which must be put on the neighborhoods $U\left(x_{1}\right), U\left(x^{1}\right)$, and $U(y)$ (used in the derivation of (2.6)).

The function $F(a \zeta, y)$ is the solution for $x^{1}$ of the equation

$$
x^{1}+E^{1} R T\left(a \zeta+x^{1}\right)-E^{1} R(y)=0 .
$$

It may be obtained by successive approximations as follows: put

$$
\begin{aligned}
x_{(0)} & =0, \quad z=E_{1} R(y), \\
x_{(m)} & =x_{(m)}(a \zeta, y)=z-E^{1} R T\left(a \zeta+x_{(m-1)}\right) \quad(m=1,2, \cdots) .
\end{aligned}
$$

Then according to the Hildebrandt-Graves implicit function theorem, $\left\{x_{(m)}\right\}$ is a Cauchy sequence in $\mathfrak{X}^{1}$ which converges to the solution of (2.4). Let $\alpha$ be a fixed real number such that $1 / 4<\alpha<1$. We choose $U\left(x_{1}\right), U\left(x^{1}\right)$, and $U(y)$ small enough so that if $U_{1}\left(x_{1}\right)$ is a fixed neighborhood in $\mathfrak{X}_{1}$ such that $\mathrm{Cl}\left(U_{1}\left(x_{1}\right)\right) \subset U\left(x_{1}\right)$, the following restrictions are satisfied $\left({ }^{7}\right)$ :

$\left(\mathrm{R}_{1}\right) \max _{a}\left\|E^{1} R L_{a \zeta}(\zeta)\right\|<\alpha$. (Condition $\left(\mathrm{P}_{1}\right)$ implies that $L_{0}=0$.)

$\left(\mathrm{R}_{2}\right)$ For each fixed $y \in U(y)$ and for all $m$,

$$
\max _{a}\left[K\left\|E^{1} R\right\|\left\|a \zeta+x_{(m)}(a \zeta, y)\right\|\right]<\frac{1}{4} .
$$

$\left(\mathrm{R}_{3}\right)$ For each fixed $y \in U(y)$ and for all $m$, $\max _{a}\left(\left\|x_{(m+1)}(a \zeta, y)-x_{(m)}(a \zeta, y)\right\|\right)^{1 / 2}$

$$
<\frac{1}{4} \max _{a}\left(\left\|x_{(m)}(a \zeta, y)-x_{(m-1)}(a \zeta, y)\right\|\right)^{1 / 2} .
$$

The maxima are taken over $a$ such that $a \zeta \in \mathrm{Cl}\left(U_{1}\left(x_{1}\right)\right)$. Equation (3.3) and condition $\left(\mathrm{P}_{2}\right)$ show that for restrictions $\left(\mathrm{R}_{2}\right)$ and $\left(\mathrm{R}_{3}\right)$ to be satisfied, it is sufficient that for all $m, a \zeta \in \mathrm{Cl}\left(U_{1}\left(x_{1}\right)\right)$, and $y \in U(y)$,

$$
\left\|x_{(m)}(a \zeta, y)\right\|<c
$$

(7) From a formal viewpoint, it would be better to introduce these restrictions on $U\left(x_{1}\right)$, $U\left(x^{1}\right)$, and $U(y)$ at the beginning of the proof of Theorem 3.1. For clarity, we state them here. 
where $c$ is a positive constant of appropriate size. The proof of the Hildebrandt-Graves theorem shows that this inequality can be satisfied by proper choice of $U\left(x_{1}\right), U\left(x^{1}\right)$, and $U(y)$. Also computation using equation (3.3) and condition $\left(\mathrm{P}_{2}\right)$ shows directly that the inequality can be satisfied. From now on, we work entirely with the newly chosen $U\left(x_{1}\right), U\left(x^{1}\right)$, and $U(y)$. The neighborhood $\mathcal{V}\left(x_{1}\right)$ is contained in $U_{1}\left(x_{1}\right)$ and $\mathcal{V}(y)$ is the neighborhood that corresponds to this $\boldsymbol{V}\left(x_{1}\right)$.

Using the same type of computation that was used in the proof of Lemma 3.3, it can be shown by induction that for each $m$ and for all $a$ such that a $\in \mathrm{Cl}\left(U_{1}\left(x_{1}\right)\right)$,

$$
\frac{d}{d a} x_{(m)}(a \zeta, y)=\lim _{\Delta a \rightarrow 0}\left\{\frac{x_{(m)}((a+\Delta a) \zeta, y)-x_{(m)}(a \zeta, y)}{\Delta a}\right\}
$$

exist. This is done by proving that for all $m$,

$$
\frac{d}{d a} x_{(m)}=-E^{1} R L_{a \zeta+x(m-1)}\left[\zeta+\frac{d}{d a} x_{(m-1)}\right] .
$$

Part $\left(\mathrm{d}_{2}\right)$ of $\left(\mathrm{P}_{7}\right)$ implies that for each $m, d x_{(m)} / d a$ is continuous in $a$. Let $I=\left[a \mid a \zeta \in \mathrm{Cl}\left(U_{1}\left(x_{1}\right)\right)\right]$ and $\mathfrak{Y}$ be the Banach space of continuous functions $f$ mapping $I$ into $\mathfrak{X}^{1}$ and having as norm $\max _{a \in I}\|f(a)\|$. We denote this norm by $\|f\|_{I}$.

Lemma 3.4. The sequence $\left\{d x_{(m)}(a \zeta, y) / d a\right\}$ is a Cauchy sequence in $\mathfrak{V}$.

Proof. Applying part $\left(\mathrm{d}_{2}\right)$ of $\left(\mathrm{P}_{7}\right)$ and restriction $\left(\mathrm{R}_{2}\right)$ to $(3.4)$, we obtain

$$
\left\|\frac{d}{d a} x_{(m+1)}\right\|_{I}<\frac{1}{4}\left(1+\left\|\frac{d}{d a} x_{(m)}\right\|_{I}\right)<\alpha\left(1+\left\|\frac{d}{d a} x_{(m)}\right\|_{I}\right) .
$$

By restriction $\left(\mathrm{R}_{1}\right),\left\|d x_{(1)} / d a\right\|_{I}<\alpha$. Hence by induction, we have for all $m$,

$$
\left\|\frac{d}{d a} x_{(m)}(a \zeta, y)\right\|_{I}<\sum_{q=1}^{q=m} \alpha^{q}<\sum_{q=1}^{\infty} \alpha^{q}=M .
$$

Direct computation using part $\left(\mathrm{d}_{2}\right)$ of $\left(\mathrm{P}_{7}\right)$, restriction $\left(\mathrm{R}_{2}\right)$, and (3.5) shows that

$$
\begin{aligned}
& \left\|\frac{d}{d a} x_{(m+1)}-\frac{d}{d a} x_{(m)}\right\|_{I} \\
& \quad<\frac{1}{4}\left\|\frac{d}{d a} x_{(m)}-\frac{d}{d a} x_{(m-1)}\right\|_{I}+\left[\left\|E^{1} R\right\|\right][K][1+M]\left[\left\|x_{(m)}-x_{(m-1)}\right\|_{I}\right] .
\end{aligned}
$$

Since $\left\{x_{(m)}\right\}$ is a Cauchy sequence in $\mathfrak{Y}$, then for all $m$ greater than some $m_{0}$, 
Hence

$$
\left(\left\|x_{(m)}-x_{(m-1)}\right\|_{I}\right)^{1 / 2}<\frac{1}{4\left[\left\|E^{1} R\right\|\right][K][1+M]} .
$$

$$
\begin{aligned}
\| \frac{d}{d a} x_{(m+1)}-\frac{d}{d a} x_{(m)} & \|_{I} \\
& <\frac{1}{4}\left\|\frac{d}{d a} x_{(m)}-\frac{d}{d a} x_{(m-1)}\right\|_{I}+\frac{1}{4}\left(\left\|x_{(m)}-x_{(m-1)}\right\|_{I}\right)^{1 / 2} .
\end{aligned}
$$

From this inequality and restriction $\left(\mathrm{R}_{3}\right)$, it follows that

$$
\sum_{m=2}^{\infty}\left[\left\|\frac{d}{d a} x_{(m)}-\frac{d}{d a} x_{(m-1)}\right\|_{I}+\left(\left\|x_{(m)}-x_{(m-1)}\right\|_{I}\right)^{1 / 2}\right]<\infty .
$$

This completes the proof of Lemma 3.4.

Now let $w(a)=\lim _{m \rightarrow \infty} d x_{(m)} / d a$. Then by standard methods, in particular by using the notion of the Riemann integral of the functions $f(a) \in \mathfrak{Y}$, it is easy to show that for all $a \in I$,

$$
\lim _{\Delta a \rightarrow 0} \frac{F[(a+\Delta a) \zeta, y]-F[a \zeta, y]}{\Delta a}=w(a) .
$$

This completes the proof of Theorem 3.1.

4. Application to integral equations. We use Theorems 2.2, 2.3, and 3.1 to obtain existence theorems for the integral equation

$$
x(s)+\int_{a}^{b} K(s, t) F[x(t)] d t=y(s)
$$

where $y(s)$ is a given real-valued continuous function on the closed interval $(a, b), K(s, t)$ is a given real-valued continuous function on the closed square $(a, b) \times(a, b)$, and $F(x)$ is a real-valued function having, for some positive $\epsilon$, a continuous second derivative in the interval $-\epsilon \leqq x \leqq \epsilon$ and such that $F(0)$ $=0$. (This last assumption does not restrict the generality.) We show that (4.1) is an example of the abstract equation (2.1) if $\mathfrak{X}$ is the Banach space (5) of continuous functions on the closed interval $(a, b)$ with norm $\max _{a \leqq s \leqq b}|x(s)|$.

Using a Taylor's expansion, we may write (4.1) in the form

$$
\begin{aligned}
x(s) & +\int_{a}^{b} K(s, t) F_{x}(0) x(t) d t \\
& +\int_{a}^{b} K(s, t)[x(t)]^{2} \int_{0}^{1}\left\{F_{x x}[\tau x(t)]\right\}\{1-\tau\} d \tau d t=y(s) .
\end{aligned}
$$

The transformation 


$$
\int_{a}^{b} K(s, t) F_{x}(0) x(t) d t
$$

acting on $x(t)$ is linear and completely continuous, and it is a straightforward computation to show that

$$
T(x)=\int_{a}^{b} K(s, t)\left\{F[x(t)]-x(t) F_{x}(0)\right\} d t
$$

satisfies conditions $\left(\mathrm{P}_{1}\right),\left(\mathrm{P}_{2}\right)$, and $\left(\mathrm{P}_{7}\right)$; for we have

$$
\begin{aligned}
T & (x+\Delta x)-T(x) \\
& =\int_{a}^{b} K(s, t)\left[F(x+\Delta x)-F(x)-\Delta x F_{x}(0)\right] d t \\
& =\int_{a}^{b} K(s, t)\left\{\Delta x\left[F_{x}(x)-F_{x}(0)\right]+(\Delta x)^{2} \int_{0}^{1}\left[F_{x x}(x+\tau \Delta x)\right][1-\tau] d \tau\right\} d t .
\end{aligned}
$$

So

$$
L_{x}(\Delta x)=\int_{a}^{b} K(s, t) \Delta x\left[F_{x}(x)-F_{x}(0)\right] d t
$$

and

$$
Q_{x}(\Delta x)=\int_{a}^{b} K(s, t)\left\{(\Delta x)^{2} \int_{0}^{1} F_{x x}(x+\tau \Delta x)(1-\tau) d \tau\right\} d t
$$

Hence we can apply the results of $\$ 2$ to (4.1).

If

$$
x(s)+\int_{a}^{b} K(s, t) F_{x}(0) x(t) d t
$$

is a nonsingular operator, then, by the Hildebrandt-Graves theorem, (4.1) has a unique local solution $x(s)$ for each sufficiently small $y(s)$. From Theorems 2.2, 2.3, and 3.1, we obtain:

THEOREM 4.1. If the multiplicity is different from zero, there exist $\epsilon_{1}, \epsilon_{2}>0$ such that for each $y(s),\|y(s)\|<\epsilon_{1}$, equation (4.1) has at least one solution $x(s)$, $\|x(s)\|<\epsilon_{2}$.

THEOREM 4.2. Suppose there is an $\epsilon_{1}>0$ such that the operator

$$
x(s)+\int_{a}^{b} K(s, t) F[x(t)] d t
$$


is 1-1 on the set of functions $x(s),\|x(s)\|<\epsilon_{1}$. Then there exist $\epsilon_{2}, \epsilon_{3}>0$ such that for each $y(s),\|y(s)\|<\epsilon_{2}$, equation (4.1) has a solution $x(s),\|x(s)\|<\epsilon_{3}$.

THEOREM 4.3. If the multiplicity $m$ is different from zero, there exist $\epsilon_{1}, \epsilon_{2}>0$ such that for a good many of the functions, $y(s),\|y(s)\|<\epsilon_{1}$, equation (4.1) has at least $|m|$ distinct solutions $x(s),\|x(s)\|<\epsilon_{2}$.

5. Application to elliptic differential equations $\left({ }^{8}\right)$. We investigate the solutions $z(x, y)$ "neighboring" a given initial solution $z_{0}(x, y)$ of the differential equation

$$
F(x, y, z, p, q, r, s, t)=\psi_{0}(x, y)
$$

where $p, q, r, s, t$ denote $z_{x}, z_{y}, z_{x x}, z_{x y}, z_{y y}$ respectively, $F$ has $\alpha$-Hölder continuous third derivatives in all its variables, and $F$ is elliptic relative to $z_{0}$, i.e.,

$$
\left[F_{s}\right]^{2}-4\left[F_{r}\right]\left[F_{t}\right]<0
$$

when $z_{0}$ and its derivatives are substituted in $F_{s}, F_{r}, F_{t}$. The solution $z_{0}$ is defined on the closure $\bar{K}$ of an open disc $K$ in the $x y$-plane and is in $E_{\alpha, 3}$. Let $\phi_{0}$ be the boundary value of $z_{0}$, i.e.,

$$
\phi_{0}=z_{0} / \bar{K}-K
$$

A solution "neighboring" $z_{0}$ is a function $z_{1}(x, y)$ whose boundary value $\phi_{1}$ is close to $\phi_{0}$ (in the topology of $e_{\alpha, 3}$ ) and which satisfies the equation

$$
F(x, y, z, p, q, r, s, t)=\psi_{1}(x, y)
$$

where $\psi_{1}$ is close to $\psi_{0}$ (in the topology of $E_{\alpha, 1}$ ).

In [3], this problem was reduced to that of investigating the solutions $(\rho, \phi)$ of an equation in $E_{\alpha, 1} \times e_{\alpha, 3}$

$$
I(\rho, \phi)+C(\rho, \phi)+T(\rho, \phi)=(\psi, \phi),
$$

where $I$ is the identity and $(\psi, \phi)$ is given. It was proved that $C$ is linear and completely continuous and that $T$ satisfies conditions $\left(\mathrm{P}_{1}\right)$ and $\left(\mathrm{P}_{2}\right)$. Here using a much simpler method, we shall prove that $T$ satisfies $\left(\mathrm{P}_{1}\right),\left(\mathrm{P}_{2}\right)$, and $\left(\mathrm{P}_{7}\right)\left({ }^{9}\right)$. That done, we can apply the theorems of $\S \S 2$ and 3 to [3.12]. We obtain then the following results for equation (5.1).

( ${ }^{8}$ We use the notation and terminology of [3]. Equations from [3] will be indicated by their numbers as given in [3] with the numbers in brackets instead of parentheses.

( ${ }^{9}$ I am indebted to the referee for pointing out this method which is much clearer and simpler than that used in [3]. This method shows that only $\alpha$-Hölder continuous third derivatives of $F$ are needed to show that $\left(\mathrm{P}_{7}\right)$ is satisfied. The referee also pointed out that no derivatives higher than the second are needed in $\$ 4$. 
If $(I+C)$ is nonsingular (i.e., if the Jacobi equation associated with (5.1) has only the zero solution [3, Lemma 3.2]), equation (5.1) has a unique local solution. If $(I+C)$ is singular, i.e. if the Jacobi equation has a nonzero solution, we apply Theorems $2.2,2.3$, and 3.1 to obtain:

THEOREM 5.1. If the multiplicity is different from zero, there exist $\epsilon_{1}, \epsilon_{2}, \epsilon_{3}>0$ such that if

$$
\left\|\psi_{1}-\psi_{0}\right\|_{\alpha, 1}<\epsilon_{1}
$$

and

$$
\left\|\phi_{1}-\phi_{0}\right\|_{\alpha, 3}<\epsilon_{2}
$$

the equation

$$
F(x, y, z, p, q, r, s, t)=\psi_{1}(x, y)
$$

has at least one solution $z_{1}(x, y)$ with boundary value $\phi_{1}$ and satisfying the inequality:

$$
\left\|z_{1}-z_{0}\right\|_{\alpha, 3}<\epsilon_{3} .
$$

Theorem 5.2 (Schauder's Theorem). If $F(x, y, z, p, q, r, s, t)$ is $a$ 1-1 mapping in some neighborhood of $z_{0}(x, y)$, there exist $\epsilon_{1}, \epsilon_{2}, \epsilon_{3}>0$ such that if

$$
\left\|\psi_{1}-\psi_{0}\right\|_{\alpha, 1}<\epsilon_{1}
$$

and

$$
\left\|\phi_{1}-\phi_{0}\right\|_{\alpha, 3}<\epsilon_{2}
$$

the equation

$$
F(x, y, z, p, q, r, s, t)=\psi_{1}(x, y)
$$

has a solution $z_{1}(x, y)$ with boundary value $\phi_{1}$ and satisfying the inequality

$$
\left\|z_{1}-z_{0}\right\|_{\alpha, 3}<\epsilon_{3} .
$$

THEOREM 5.3. If the multiplicity $m$ is different from zero, there exist $\epsilon_{1}, \epsilon_{2}$, $\epsilon_{3}>0$ such that for a good many of the pairs $\left(\psi_{1}, \phi_{1}\right)$,

$$
\left\|\psi_{1}-\psi_{0}\right\|_{\alpha, 1}<\epsilon_{1}
$$

and

$$
\left\|\phi_{1}-\phi_{0}\right\|_{\alpha, 3}<\epsilon_{2}
$$

the equation 


$$
F(x, y, z, p, q, r, s, t)=\psi_{1}(x, y)
$$

has at least $|m|$ distinct solutions $z_{1}$ with boundary value $\phi_{1}$ and satisfying the inequality

$$
\left\|z_{1}-z_{0}\right\|_{\alpha, 3}<\epsilon_{3}
$$

It remains to show that $T$ satisfies conditions $\left(\mathrm{P}_{1}\right),\left(\mathrm{P}_{2}\right)$, and $\left(\mathrm{P}_{7}\right)$. The transformation $T(\rho, \phi)$ was defined in $[3$, pp. 114-118] as

$$
T(\rho, \phi)=\left(R_{2}^{(1)}(\rho, \phi), 0\right)+\left(R^{(2)}(\rho, \phi), 0\right)
$$

where

$$
\begin{aligned}
R_{2}^{(1)}(\rho, \phi)+ & R^{(2)}(\rho, \phi) \\
= & F\left(x, y, z_{0}+w, p_{0}+w_{x}, q_{0}+w_{y}, r_{0}+w_{x x}, s_{0}+w_{x y}, t_{0}+w_{y y}\right) \\
& \quad-w F_{z}^{0}-w_{x} F_{p}^{0}-w_{y} F_{q}^{0}-\rho
\end{aligned}
$$

where

$$
\rho=\rho(x, y)=w_{x x} F_{r}^{0}+w_{x y} F_{s}^{0}+w_{y y} F_{t}^{0}
$$

and the superscript zero means that the arguments of the functions are $x, y, z_{0}, p_{0}, q_{0}, r_{0}, s_{0}, t_{0}$.

Since $w$ and its derivatives are linear functions of $(\rho, \phi)$ and $F\left(x, y, z_{0}, p_{0} q_{0}, r_{0}\right.$, $\left.s_{0}, t_{0}\right) \equiv 0$, it is clear that $T(0)=0$, i.e., condition $\left(\mathrm{P}_{1}\right)$ is satisfied.

Now let $(\rho, \phi)$ and $\left(\rho^{\prime}, \phi^{\prime}\right)$ be two points in $E_{\alpha, 1} \times e_{\alpha, 3}$. We indicate arguments by superscripts in this way:

$$
\begin{aligned}
w & =w(\rho, \phi), \\
w^{\prime} & =w\left(\rho^{\prime}, \phi^{\prime}\right), \\
F & =F\left(x, y, z_{0}+w, p_{0}+w_{x}, q_{0}+w_{y}, r_{0}+w_{x x}, s_{0}+w_{x y}, t_{0}+w_{y y}\right), \\
F^{\prime} & =F\left(x, y, z_{0}+w^{\prime}, p_{0}+w_{x}^{\prime}, q_{0}+w_{y}^{\prime}, r_{0}+w_{x x}^{\prime}, s_{0}+w_{x y}^{\prime}, t_{0}+w_{y y}^{\prime}\right), \\
F^{\tau}=F\left[x, y, z_{0}+w+\tau\left(w^{\prime}-w\right), p_{0}+w_{x}+\tau\left(w^{\prime}-w\right)_{x},\right. & q_{0}+w_{y}+\tau\left(w^{\prime}-w\right)_{y}, r_{0}+w_{x x}+\tau\left(w^{\prime}-w\right)_{x x}, \\
& \left.s_{0}+w_{x y}+\tau\left(w^{\prime}-w\right)_{x y}, t_{0}+w_{y y}+\tau\left(w^{\prime}-w\right)_{y y}\right] .
\end{aligned}
$$

Then 


$$
\begin{aligned}
R_{2}^{(1)}\left(\rho^{\prime}, \phi^{\prime}\right)+R & \left(\rho^{\prime}, \phi^{\prime}\right)-R_{2}^{(1)}(\rho, \phi)-R^{(2)}(\rho, \phi) \\
= & \left(F^{\prime}-F\right)-\left[\left(w^{\prime}-w\right) F_{z}^{0}+\left(w^{\prime}-w\right)_{x} F_{p}^{0}+\left(w^{\prime}-w\right)_{y} F_{q}^{0}\right] \\
& -\left[\left(w^{\prime}-w\right)_{x x} F_{r}^{0}+\left(w^{\prime}-w\right)_{x y} F_{s}^{0}+\left(w^{\prime}-w\right)_{y y} F_{t}^{0}\right] \\
= & \left(w^{\prime}-w\right) F_{z}+\left(w^{\prime}-w\right)_{y} F_{p}+\left(w^{\prime}-w\right)_{y} F_{q}+\left(w^{\prime}-w\right)_{x x} F_{r} \\
& +\left(w^{\prime}-w\right)_{x y} F_{s}+\left(w^{\prime}-w\right)_{y y} F_{t} \\
& +\int_{0}^{1}\left[\left(w^{\prime}-w\right)^{2} F_{z z}^{\tau}+2\left(w^{\prime}-w\right)\left(w^{\prime}-w\right)_{x} F_{z p}^{\tau}+\cdots\right](1-\tau) d \tau \\
& -\left[\left(w^{\prime}-w\right)_{z}^{0}+\left(w^{\prime}-w\right)_{x} F_{p}^{0}+\left(w^{\prime}-w\right)_{y} F_{q}^{0}\right] \\
& -\left[\left(w^{\prime}-w\right)_{x x} F_{r}^{0}+\left(w^{\prime}-w\right)_{x y} F_{s}^{0}+\left(w^{\prime}-w\right)_{y y} F_{t}^{0}\right] \\
= & \left(w^{\prime}-w\right)\left(F_{z}-F_{z}^{0}\right)+\left(w^{\prime}-w\right)_{x}\left(F_{p}-F_{p}^{0}\right) \\
& +\left(w^{\prime}-w\right)_{y}\left(F_{q}-F_{q}^{0}\right)+\left(w^{\prime}-w\right)_{x x}\left(F_{r}-F_{r}^{0}\right) \\
& +\left(w^{\prime}-w\right)_{x y}\left(F_{s}-F_{s}^{0}\right)+\left(w^{\prime}-w\right)_{y y}\left(F_{t}-F_{t}^{0}\right) \\
& +\int_{0}^{1}\left[\left(w^{\prime}-w\right)^{2} F_{z z}^{\tau}+2\left(w^{\prime}-w\right)\left(w^{\prime}-w\right)_{x} F_{z p}^{\tau}+\cdots\right](1-\tau) d \tau .
\end{aligned}
$$

Applying [3, Lemma 3.1] to this last expression shows that $T$ satisfies condition $\left(\mathrm{P}_{7}\right)$. The integral is the $Q_{x}(\Delta x)$ and the sum of the remaining terms is the $L_{x}(\Delta x)$. Applying the mean value theorem to $F_{z}-F_{z}^{0}$ and other differences of this form in (5.2) and again using [3, Lemma 3.1] shows that $T$ satisfies condition $\left(\mathrm{P}_{2}\right)$. It is necessary to assume that $F$ has $\alpha$-Hölder continuous third derivatives because norms in an $E_{\alpha, 1}$ are considered.

\section{BIBLIOGRAPHY}

1. P. Alexandroff and H. Hopf, Topologie 1, Berlin, 1935.

2. J. Cronin, Branch points of solutions of equations in Banach space, Trans. Amer. Math. Soc. vol. 69 (1950) pp. 208-231.

3. - The existence of multiple solutions of elliptic differential equations, Trans. Amer. Math. Soc. vol. 68 (1950) pp. 105-131.

4. T. H. Hildebrandt and L. M. Graves, Implicit functions and their differentials in general analysis, Trans. Amer. Math. Soc. vol. 29 (1927) pp. 127-153.

5. C. C. MacDuffee, Vectors and matrices, Carus Mathematical Monographs, No. 7, Mathematical Association of America, 1943.

6. F. Riesz, Über lineare Funktionalgleichungen, Acta Math. vol. 41 (1918) pp. 71-98.

7. A. Sard, The measure of the critical values of differentiable maps, Bull. Amer. Math. Soc. vol. 48 (1942) pp. 883-890.

8. J. Schauder, Über den Zusammenhang zwischen der Eindeutigkeit und Lösbarkeit partieller Differentialgleichungen zweiter Ordnung von elliptischen Typus, Math. Ann. vol. 106 (1932) pp. 661-721.

UNIVERSITY OF MICHIGAN, ANN ARbor, Mich.

Air Force Cambridge Research Center, Cambridge, Mass. 